\title{
A NEW PROOF OF THE DOYEN-WILSON THEOREM
}

\author{
D. R. STINSON
}

(Received 10 November 1987)

Communicated by Louis Caccetta

\begin{abstract}
We describe several recursive constructions for designs which use designs with "holes". As an application, we give a short new proof of the Doyen-Wilson Theorem.
\end{abstract}

1980 Mathematics subject classification (Amer. Math. Soc.) (1985 Revision): 05 B 05, 05 B 07.

\section{Introduction}

A pairwise balanced design (or, PBD) is a pair $(X, \mathscr{A})$, such that $X$ is a set of elements (called points) and $\mathscr{A}$ is a set of subsets of $X$ (called blocks), each of cardinality at least two, such that every unordered pair of points is contained in a unique block of $\mathscr{A}$. If $v$ is a positive integer and $K$ is a set of positive integers, each of which is greater than or equal to 2 , then we say that $(X, \mathscr{A})$ is a $(v, K)$-PBD if $|X|=v$, and $|A| \in K$ for every $A \in \mathscr{A}$. The integer $v$ is called the order of the PBD.

Using this notation, we can define a Steiner triple system of order $v$, which we denote $\operatorname{STS}(v)$, to be a $(v,\{3\})$-PBD. It is of course well-known that an STS $(v)$ exists if and only if $v \equiv 1$ or 3 modulo 6 .

Let $(X, \mathscr{A})$ be a PBD. If a set of points $Y \subseteq X$ has the property that, for any $A \in \mathscr{A}$, either $|Y \cap A| \leq 1$ or $A \subseteq Y$, then we say that $Y$ is a subdesign or flat of the PBD. The order of the subdesign is $|Y|$. The subdesign $Y$ is proper if $Y \neq X$. If $Y$ is a subdesign, then we can delete all blocks $A \subseteq Y$ and

(C) 1989 Australian Mathematical Society $0263-6115 / 89 \$ A 2.00+0.00$ 
replace them by a single block, $Y$, and the result is a PBD. Also, any block or point of a PBD is itself a subdesign.

In the case of Steiner triple systems, it is easy to see that if an STS $(v)$ contains an $\operatorname{STS}(w)$ as a proper subdesign, then $v \geq 2 w+1$. Of course, $v \equiv 1$ or 3 modulo 6 and $w \equiv 1$ or 3 modulo 6 . In 1973, Doyen and Wilson showed in [3] that these necessary conditions were sufficient.

DOYEN-WILson THEOREM. There exists an STS $(v)$ which contains an STS $(w)$ as a proper subdesign if and only if $v \geq 2 w+1, v \equiv 1$ or 3 modulo 6 , and $w \equiv 1$ or 3 modulo 6 .

More recently, a very different proof of this result has been given by Stern and Lenz [15], using graph-theoretic methods. Generalizations of the DoyenWilson theorem have been studied in [14] and [7].

In this paper, we give yet another proof of this theorem, which is recursive and completely design-theoretic in nature. This proof depends on some new constructions utilizing designs with "holes", which we describe in the next section. Once we have developed the necessary machinery, the proof of the Doyen-Wilson Theorem which we present is very brief. This is done in Section 3.

\section{Designs with holes}

In this section, we review some well-known design constructions using various types of designs with "holes", and present some new generalizations of these constructions. This section is intended to be a leisurely exposition, so that the concepts we discuss can be made as clear as possible.

First, we define a useful generalizations of a PBD, called a group-divisible design. A group-divisible design (or, GDD), is a triple $(X, \mathscr{G}, \mathscr{A})$, which satisfies the following properties:

(1) $\mathscr{G}$ is a partition of $X$ into subsets called groups;

(2) $\mathscr{A}$ is a set of subsets of $X$ (called blocks) such that a group and a block contain at most one common point;

(3) every pair of points from distinct groups occurs in a unique block.

The group-type of a $\operatorname{GDD}(X, \mathscr{G}, \mathscr{A})$ is the multiset $\{|G|: G \in \mathscr{G}\}$. We usually use an "exponential" notation to describe group-types: a group-type $1^{i} 2^{j} 3^{k} \ldots$ denotes $i$ occurrences of $1, j$ occurrences of 2 , etc. As with PBDs, we will say that a GDD is a $K$-GDD if $|A| \in K$ for every $A \in \mathscr{A}$. 
A parallel class in a GDD or PBD is a set of blocks that form a partition of the point set. A GDD or PBD is resolvable if the block set can be partitioned into parallel classes.

Now, we define the idea of a GDD with a hole. Informally, an incomplete GDD, or IGDD, is a GDD from which a sub-GDD is missing (this is the "hole"). We give a formal definition. An IGDD is a quadruple $(X, Y, \mathscr{G}, \mathscr{A})$ which satisfies the following properties:

(1) $X$ is a set of points, and $Y \subseteq X$;

(2) $\mathscr{G}$ is a partition of $X$ into groups;

(4) $\mathscr{A}$ is a set of blocks, each of which intersects each group in at most one point;

(5) no block contains two members of $Y$;

(6) every pair of points $\{x, y\}$ from distinct groups, such that at least one of $x, y$ is in $X \backslash Y$, occurs in a unique block of $\mathscr{A}$.

We say that an $\operatorname{IGDD}(X, Y, \mathscr{G}, \mathscr{A})$ is a $K$-IGDD if $|A| \in K$ for every block $A \in \mathscr{A}$. The type of the IGDD is defined to be the multiset of ordered pairs $\{(|G|,|G \cap Y|): G \in \mathscr{G}\}$. As with GDDs, we shall use an exponential notation to describe types. Note that if $Y=\varnothing$, then the IGDD is a GDD.

We have already defined PBDs with subdesigns. If we allow the subdesign to be missing (i.e., a hole), we have an incomplete PBD, as follows. An incomplete PBD (or (IPBD) is a triple $(X, Y, \mathscr{A})$, where $X$ is a set of points, $Y \subseteq X$, and $\mathscr{A}$ is a set of blocks which satisfies the following properties:

(1) for any $A \in \mathscr{A},|A \cap Y| \leq 1$;

(2) any two points $x, y$, not both in $Y$, occur in a unique block.

Hence, $Y$ is the hole. Note that $(X, Y, \mathscr{A})$ is an IPBD if and only if $(X, \mathscr{A} \cup$ $\{Y\})$ is a PBD. We say that $(X, Y, \mathscr{A})$ is a $(v, w ; K)$-IPBD if $|X|=v,|Y|=w$, and $|A| \in K$ for every $A \in \mathscr{A}$. Of course, we can fill in the hole of an IPBD, as follows.

Construction 2.1. Suppose $(X, Y, \mathscr{A})$ is an IPBD and $(Y, \mathscr{B})$ is a PBD. Then $(X, \mathscr{A} \cup \mathscr{B})$ is a PBD.

We can obtain IPBDs from IGDDs by filling in groups. The following construction was first stated in a general form in [9, Construction 4.1], although it was earlier applied in various special cases (see, for example, [2], [5], and [4]).

CONSTRUCTION 2.2. FILLING IN GROUPS. Let $K$ be a set of positive integers, and let $a \geq 0$. Suppose that there exists a $K$-IGDD of type $\left\{\left(t_{1}, u_{1}\right),\left(t_{2}, u_{2}\right)\right.$, $\left.\ldots,\left(t_{n}, u_{n}\right)\right\}$, and suppose $\left(t_{i}+a, u_{i}+a ; K\right)$-IPBDs exist for $1 \leq i \leq n$. Then there exists a $(t+a, u+a ; K)-\mathrm{IPBD}$, where $u=\sum u_{i}$ and $t=\sum t_{i}$. 
The special case when we start with a GDD (that is, $u_{1}=\cdots=u_{n}=0$ ) and when we fill in the hole at the end is the construction Wilson calls "adjoining subdesigns" [16].

Construction 2.3: ADJoINING SUBDEsigns. Let $K$ be a set of positive integers, and let $a \geq 0$. Suppose that the following designs exist: a $K$-GDD of type $\left\{t_{1}, t_{2}, \ldots, t_{n}\right\} ;\left(t_{i}+a, a ; K\right)$-IPBDs, for $1 \leq i \leq n$; and an $(a, K)-\mathrm{PBD}$. Then there exists a $(t+a, K)-\mathrm{PBD}$, where $t=\sum t_{i}$, containing subdesigns of order $t_{i}+a,(1 \leq i \leq n)$, and order $a$.

One of our main tools in the proof of the Doyen-Wilson Theorem is a more powerful method of filling in groups. First, we need to define a more general type of incomplete PBD. PBDs can contain many different subdesigns, which in turn can intersect in further subdesigns. These subdesigns will form a lattice. We are interested in the situation when the lattice is a square. That is, we have two subdesigns, of given sizes, which intersect in a third subdesign of a given size. However, as before, the subdesigns need not be present, that is, we allow holes. We will refer to the designs as $\triangle$-IPBDs, in order to suggest the lattice structure. Based on this informal discussion, we give a formal definition. An incomplete $\diamond-\mathrm{PBD}$ is a tuple $\left(X, Y_{1}, Y_{2}, \mathscr{A}\right)$, where $Y_{1} \subseteq X$, $Y_{2} \subseteq X$, and $\mathscr{A}$ is a set of blocks such that every pair of points $\{x, y\}$ occurs in a unique block, unless $\{x, y\} \subset Y_{1}$ or $\{x, y\} \subseteq Y_{2}$, in which case the pair occurs in no block. We say that the $\checkmark$-IPBD is a $\left(v ; w_{1}, w_{2} ; w_{3} ; K\right)$ - $\diamond$-IPBD if $|X|=v,\left|Y_{1}\right|=w_{1},\left|Y_{2}\right|=w_{2},\left|Y_{1} \cap Y_{2}\right|=w_{3}$, and $|A| \in K$ for every $A \in \mathscr{A}$.

ExAmple. $A(15 ; 7,7 ; 3 ;\{3\})$ - $\diamond$-IPBD. $X=\{1,2, \ldots, 15\}, Y_{1}=\{1,2,3$, $4,13,14,15\}$, and $Y_{2}=\{5,6,7,8,13,14,15\}$. The blocks are $\{1,5,9\},\{2,6,9\},,\{3,7,9\},\{4,8,9\},\{1,6,10\},\{2,7,10\},\{3,8,10\}$, $\{4,5,10\},\{1,7,11\},\{2,8,11\},\{3,5,11\},\{4,6,11\},\{1,8,12\},\{2,5,12\}$, $\{3,6,12\},\{4,7,12\},\{9,10,13\},\{11,12,13\},\{9,11,14\},\{10,12,14\}$, $\{9,12,15\},\{10,11,15\}$.

The holes of $\triangle$-IPBD can be filled in various ways. We have the following simple observations.

Construction 2.4. Suppose $\left(X, Y_{1}, Y_{2}, \mathscr{A}\right)$ is a $\diamond$-IPBD, and that $\left(Y_{1}, Y_{1} \cap Y_{2}, \mathscr{B}\right)$ and $\left(Y_{2}, Y_{1} \cap Y_{2}, \mathscr{C}\right)$ are IPBDs. Then $\left(X, Y_{1}, \mathscr{A} \cup \mathscr{C}\right),\left(X, Y_{2}, \mathscr{A}\right.$ $\cup \mathscr{B})$, and $\left(X, Y_{1} \cap Y_{2}, \mathscr{A} \cup \mathscr{B} \cup \mathscr{C}\right)$ are IPBDs.

REMARK. If we fill in all but one group of an IGDD by the method of Construction 2.2, we obtain a $\diamond$-IPBD. 
Our main application of $\triangle$-IPBDs involves using them to fill in the groups of IGDDs. This is a generalization of Construction 2.2.

CONSTRUCTION 2.5: GENERALIZED FILLING IN GROUPS. Let $K$ be a set of positive integers, and let $b \geq a \geq 0$. Suppose that the following designs exist:

(1) a $K$-IGDD of type $\left\{\left(t_{1}, u_{1}\right),\left(t_{2}, u_{2}\right), \ldots,\left(t_{n}, u_{n}\right)\right\}$;

(2) a $\left(t_{i}+b ; u_{i}+a, b ; a ; K\right)-\diamond-\mathrm{IPBD}$, for $1 \leq i \leq n$; and

(3) a $(b, a ; K)$-IPBD.

Then there exists a $(t+b, u+a ; K)$-IPBD, where $u=\sum u_{i}$ and $t=\sum t_{i}$.

REMARK. R. Rees (private communication) has observed that we can construct the desired design under slightly weaker hypotheses, as follows.

CONSTRUCTION 2.6: GENERALIZED FILLING IN GROUPS. Let $K$ be a set of positive integers, and let $b \geq a \geq 0$. Suppose that the following designs exist:

(1) a $K$-IGDD of type $\left\{\left(t_{1}, u_{1}\right),\left(t_{2}, u_{2}\right), \ldots,\left(t_{n}, u_{n}\right)\right\}$;

(2) a $\left(t_{i}+b ; u_{i}+a, b ; a ; K\right)-\diamond-\mathrm{IPBD}$, for $1 \leq i \leq n-1$; and

(3) a $\left(t_{i}+b ; u_{i}+a\right)$-IPBD.

Then there exists a $(t+b, u+a ; K)$-IPBD, where $u=\sum u_{i}$ and $t=\sum t_{i}$.

As applications of the "filling in groups" technique, we mention a family of constructions which are called the product constructions. These are based on filling in the groups of (incomplete) transversal designs, which we now define. A transversal design $\operatorname{TD}(k, n)$ is a $\{k\}$-GDD of type $n^{k}$. It is well-known that a $\operatorname{TD}(k, n)$ is equivalent to $k-2$ mutually orthogonal Latin squares (MOLS) of order $n$. We also define a $\operatorname{TD}(k, n)-\operatorname{TD}(k, m)$ (an incomplete transversal design) to be a $\{k\}$-IGDD of group-type $(n, m)^{k}$.

The following construction is referred to as the singular indirect product, or SIP [9, Theorem 4.5] (see also [8]).

CONSTRUCTION 2.7: SINGULAR INDIRECT PRODUCT. Suppose $K$ is a set of positive integers and $u \in K$; suppose $v, w$, and $a$ are integers such that $0 \leq a \leq w \leq v$; and suppose that there exists a $\operatorname{TD}(u, v-a)-\operatorname{TD}(u, w-a)$ and a $(v, w ; K)$-IPBD. Then, a $(u(v-a)+a, u(w-a)+a ; K)$-IPBD exists.

If we let $w=a$ in the singular indirect product, we obtain the singular direct product, or SDP.

Construction 2.8: singular diRect PRoduct. Suppose $K$ is a set of positive integers and $u \in K$. Suppose $v$ and $w$ are non-negative integers such that $w \leq v$, there exists a $\operatorname{TD}(u, v)$, and there is a $(v, w ; K)$-IPBD. Then there is a $(u(v-w)+w, w ; K)$-IPBD. If further, there exists a $(w, K)$-PBD, then there exists a $(u(v-w)+w ; v ; K)$-IPBD. 
At this point we want to state a generalized singular indirect product (GSIP), using the 0 -IPBDs to fill in groups of an ITD.

CONSTRUCTION 2.9: GENERALIZED SINGULAR INDIRECT PRODUCT. Let $K$ be a set of positive integers and $u \in K$, and let $v, w, a$, and $b$ be non-negative integers such that $0 \leq b-a \leq v-w, a \leq w \leq v$, and $a \leq b \leq v$. Suppose that the following designs exist: a $\operatorname{TD}(u, v-b)-\operatorname{TD}(u, w-a), a(v ; w, b ; a ; K)$ $\checkmark$-IPBD; and a $(b, a ; K)$-IPBD. Then there exists a $(u(v-b)+b, u(w-a)+$ $a ; K)$-IPBD.

REMARK. When $b=a$, GSIP becomes SIP.

If we are to fill in groups of IGDDs, we need methods of building IGDDs. Our basic construction is a recursive one. We refer to it as the "Fundamental IGDD Construction". A variation of this construction is presented in [12].

CONSTRUCTION 2.10: FUndamental IGDD construction. Suppose $(X, Y, \mathscr{G}, \mathscr{A})$ is an IGDD, which we call the master IGDD. Let $t, s: X \rightarrow$ $\mathbf{Z}^{+} \cup\{0\}$ be functions such that $t(x) \leq s(x)$, for every $x \in X$ (we refer to $t$ and $s$ as weightings). For every $x \in X$, let $S(x)$ be a set of cardinality $s(x)$ and let $T(x)$ be a set of cardinality $t(x)$, where $T(x) \subseteq S(x)$. Also, suppose that $S(x) \cap S(y)=\varnothing$ if $x \neq y$. For any subset $Y \subseteq X$, denote $S(Y)$ (resp. $T(Y)$ ) to be $\bigcup_{x \in Y} S(x)$ (respectively $\bigcup_{x \in Y} T(x)$ ). Now, for every block $A \in \mathscr{A}$, suppose that we have an IGDD.

$$
(S(A), T(A),\{S(x): x \in A\}, \mathscr{B}(A)) .
$$

Also, suppose that we have an IGDD,

$$
(S(Y), T(Y),\{S(G \cap Y): G \in \mathscr{G}\}, \mathscr{B}(Y)) .
$$

Then

is an IGDD.

$$
\left(S(X), T(X),\{S(G): G \in \mathscr{G}\}, \bigcup_{A \in \mathscr{A}} \mathscr{B}(A) \cup \mathscr{B}(Y)\right)
$$

When the master IGDD is a GDD, we obtain a construction which was presented by Mullin et al. in [9, Construction 4.4].

Construction 2.11. Suppose $(X, \mathscr{G}, \mathscr{A})$ is a GDD, and let $t, s: X \rightarrow$ $\mathrm{Z}^{+} \cup\{0\}$ be functions such that $t(x) \leq s(x)$, for every $x \in X$. For every $x \in X$, let $S(x)$ be a set of cardinality $s(x)$ and let $T(x)$ be a set of cardinality $t(x)$, where $T(x) \subseteq S(x)$. Also, suppose $S(x) \cap S(y)=\varnothing$ if $x \neq y$. For any subset $Y \subseteq X$, define $S(Y)$ (resp. $T(Y))$ to be $\bigcup_{x \in Y} S(x)$ (resp. $\bigcup_{x \in Y} T(x)$ ). For every block $A \in \mathscr{A}$, suppose that we have an IGDD,

$$
(S(A), T(A),\{S(x): x \in A\}, \mathscr{B}(A)) .
$$


Then

$$
\left(S(X), T(X),\{S(G): G \in \mathscr{G}\}, \bigcup_{A \in \mathscr{A}} \mathscr{B}(A)\right)
$$

is an IGDD.

As an immediate corollary of this construction we obtain the Fundamental Construction for GDDs [16].

Construction 2.12: Fundamental GDD construction. Suppose $(X, \mathscr{G}, \mathscr{A})$ is a GDD, and let $s: X \rightarrow \mathbf{Z}^{+} \cup\{0\}$ be a function. For every $x \in X$, let $S(x)$ be a set of cardinality $s(x)$. Also, suppose $S(x) \cap S(y)=\varnothing$ if $x \neq y$. For any subset $Y \subseteq X$, define $S(Y)$ to be $\bigcup_{x \in Y} S(x)$. For every block $A \in \mathscr{A}$, suppose that we have a GDD,

$$
(S(A),\{S(x): x \in A\}, \mathscr{B}(A)) .
$$

Then

$$
\left(S(X),\{S(G): G \in \mathscr{G}\}, \bigcup_{A \in \mathscr{A}} \mathscr{B}(A)\right)
$$

is a GDD.

\section{A new proof of the Doyen-Wilson Theorem}

We present two lemmata which suffice to prove the Doyen-Wilson Theorem, except for a handful of special cases which are dealt with by means of the product constructions and ad hoc methods.

LeMma 3.1. For all $w \equiv 3$ modulo 6 , and for all $v \equiv 1$ or 3 modulo 6 , $2 w+1 \leq v \leq 3 w$, there exists an $\operatorname{STS}(v)$ containing an $\operatorname{STS}(w)$. For all $w \equiv 1$ modulo $6, w \geq 25$, and for all $v \equiv 1$ or 3 modulo $6,2 w+1 \leq v \leq 3 w-6$, there exist an $\operatorname{STS}(v)$ containing an $\operatorname{STS}(w)$

Proof. Suppose we have a resolvable $\{3\}$-GDD of type $g^{u}$ (these designs have been studied in [10], [1], and [6]). Adjoin infinite points to $t$ parallel classes (where $0 \leq t \leq g(u-1) / 2$ ), constructing a $\{3,4\}$-GDD of type $g^{u} t^{1}$. Now, give the points of the original GDD weight $(2,1)$, give the $t$ infinite points weight $(2,0)$, and apply the IGDD construction. Since every block of size 4 hits the group of size $t$, we require only IGDDs of type $(2,1)^{3}$ and $(2,1)^{3} 2^{1}$. There exist $\{3\}$-IGDDs of these two types: a $\{3\}$-IGDD of type $(2,1)^{3}$ is just a $\{3\}$-GDD of type $2^{3}$ with a block removed; and a $\{3\}$-IGDD of type $(2,1)^{3} 2^{1}$ is just a $\{3\}$-GDD of type $2^{4}$ with a block removed (these are 
constructed by removing a point from an STS(7) and STS(9), respectively). In this way, we produce a $\{3\}$-IGDD of type $(2 g, g)^{u}(2 t)^{1}$.

If we take $g=1$, we start with a resolvable $\{3\}$-GDD of type $1^{u}$, which exists for all $u \equiv 3$ modulo 6 [10] (these are just resolvable STS $(u)$, or Kirkman triple systems). The construction builds $\{3\}$-IGDD of type $(2,1)^{u}(2 t)^{1}$, for all $0 \leq t \leq(u-1) / 2$. If we take $t \equiv 0$ or 1 modulo 3 , then we can adjoin a point at infinity $(b=1, a=0)$, filling in $\operatorname{STS}(3)$ and STS $(2 t+1)$. Hence, we end up with an $\operatorname{IPBD}(2 u+2 t+1, u ;\{3\})$. Filling in the hole, we have an $\operatorname{STS}(2 u+2 t+1)$ containing an $\operatorname{STS}(u)$. That is, for all $w \equiv 3$ modulo 6 , we can construct an $\operatorname{STS}(v)$ containing an STS $(w)$ for all $v \equiv 1$ or 3 modulo 6 , $2 w+1 \leq v \leq 3 w$.

If we take $g=6$, then we start with a resolvable $\{3\}$-GDD of type $6^{u}$, which exists for all $u \geq 4[11,1]$. Adjoining infinite points, we obtain a $\{3\}$-IGDD of type $(12,6)^{u}(2 t)^{1}, 0 \leq t \leq 3(u-1)$. Let $t \equiv 0$ or 2 modulo 3 , and adjoin 3 points at infinity $(b=3, a=1)$, filling in a $\diamond$-IPBD $(15 ; 7,3 ; 1 ;\{3\})$, an $\operatorname{IPBD}(2 t+3,3 ;\{3\})$, and an $\operatorname{IPBD}(3,1 ;\{3\})$. We end up with an $\operatorname{IPBD}(12 u+2 t+3,6 u+1 ;\{3\})$. The hole can then be filled in with an $\operatorname{STS}(6 u+1)$. So, for all $w \equiv 1$ modulo $6, w \geq 25$, we can construct an STS $(v)$ containing an STS $(w)$ for all $v \equiv 1$ or 3 modulo $6,2 w+1 \leq v \leq 3 w-6$.

LeMMA 3.2. For $w \equiv 1$ modulo $6, w \neq 13$, and for all $v \equiv 1$ or 3 modulo $6,3 w-2 \leq v \leq 5 w-4$, there exists an $\operatorname{STS}(v)$ containing an $\operatorname{STS}(w)$. For $w \equiv 3$ modulo 6 , and for all $v \equiv 1$ or 3 modulo $6,3 w-2 \leq v \leq 5 w-6$, there exists an $\operatorname{STS}(v)$ containing an $\operatorname{STS}(w)$.

Proof. Start with a $\operatorname{TD}(4, m)$, where $m \equiv 0$ or 1 modulo $3, m \neq 6$. Assign weight 2 to points in the first three groups, and weights 0,2 , and 4 to the points in the last group. Apply the GDD construction filling in \{3\}-GDDs of types $2^{3}, 2^{4}$, and $2^{3} 4^{1}$ (these are obtained respectively from an STS(7), STS(9), and by completing a one-factorization of $K_{6}$ ). We construct a $\{3\}$ GDD of type $(2 m)^{3}(2 t)^{1}$, for all $0 \leq t \leq 2 m$. If $t \equiv 0$ or 1 modulo 3 , then we can add on one point at infinity $(a=b=1)$ and fill $\operatorname{STS}(2 t+1)$ and $\operatorname{STS}(2 m+1)$. For $w \equiv 1$ modulo $6, w \neq 13$, this allows us to construct an $\operatorname{STS}(v)$ containing an $\operatorname{STS}(w)$ for all $3 w-2 \leq v \leq 5 w-4$. For $w \equiv 3$ modulo 6, this allows us to construct an STS $(v)$ containing an STS $(w)$ for all $3 w-2 \leq v \leq 5 w-6$.

We have only a few special cases to handle before we can prove the main result. These are described in Table 1 . We note that a $\operatorname{TD}(3, m)-\operatorname{TD}(3, n)$ exists whenever $m \geq 2 n$.

Now we can prove the Doyen-Wilson Theorem. 


\section{Table 1}

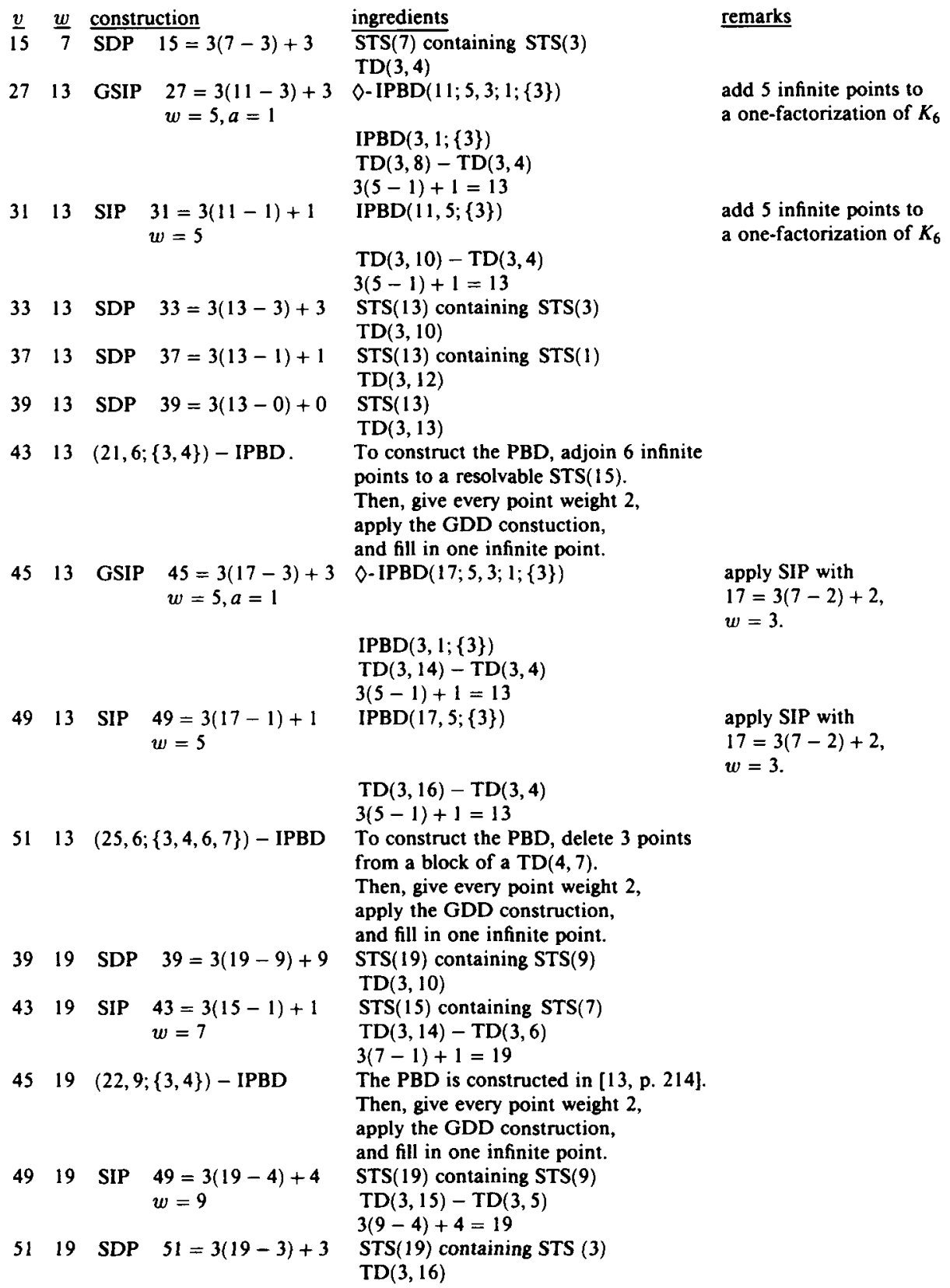


TheOREM. For all $w \equiv 1$ or 3 modulo 6 , and for all $v \equiv 1$ or 3 modulo 6 , $v \geq 2 w+1$, there exists an STS $(v)$ containing an STS $(w)$ as a subsystem.

Proof. If $w=3$, the theorem is true since any block of an STS $(v)$ is a sub-STS $(3)$; so henceforth we assume $w \geq 7$.

First, we prove the theorem is true for $2 w+1 \leq v<4 w+3$, as follows. If $w \equiv 3$ modulo $6, w \geq 9$, then the theorem is true for $2 w+1 \leq v \leq 3 w$ by Lemma 3.1, and for $3 w-2 \leq v \leq 5 w-6$ by Lemma 3.2. If $w \equiv 1$ modulo $6 w \geq 25$, the theorem is true for $2 w+1 \leq v \leq 3 w-6$ by Lemma 3.1, and for $3 w-2 \leq v \leq 5 w-4$ by Lemma 3.2. For $w=7,13$, or 19 , the theorem is true for $2 w+1 \leq v \leq 4 w-1$, by Lemma 3.2 and Table 1 .

Now, we prove the theorem by induction on the ratio $[(v+1) /(w+1)]$ (where $[x]$ denotes the largest integer not exceeding $x$ ). By the above observations, the theorem is true when the ratio $[(v+1) /(w+1)]=2$ or 3 , so we can assume $[(v+1) /(w+1)] \geq 4$. By our induction hypothesis, there exists an $\operatorname{STS}(v)$ containing an $\operatorname{STS}(2 w+1)$ and there is an $\operatorname{STS}(2 w+1)$ containing an STS $(w)$. Hence, there exists an STS $(v)$ containing an STS $(w)$. This proves the theorem.

\section{References}

[1] A. M. Assaf and A. Hartman, 'Resolvable group divisible designs with block size 3', Ann. Discrete. Math., to appear.

[2] A. E. Brouwer, 'Optimal packings of $K_{4}$ 's into a $K_{n}$ ', J. Combin. Theory Ser. A 26 (1979), 278-297.

[3] J. Doyen and R. M. Wilson, 'Embeddings of Steiner triple systems', Discrete Math. 5 (1973), 229-239.

[4] J. D. Horton, R. C. Mullin, and R. G. Stanton, 'Minimal coverings of pairs by quadruples', Proc. 2nd Louisiana Conf. on Combinatorics, Graph Theory and Computing, Baton Rouge, La. (1971), 495-516.

[5] J. G. Kalbfleisch, R. C. Mullin, and R. G. Stanton, 'Covering and packing designs', Proc. 2nd Chapel Hill Conf. on Combinatorial Math. (1970), 428-456.

[6] E. Mendelsohn and S. Hao, 'A construction of resolvable group divisible designs with block size three', Ars Combin. 24 (1987), 39-43.

[7] E. Mendelsohn and A. Rosa, 'Embedding maximal packings of triples', Congr. Numer. 40 (1983), 235-247.

[8] R. C. Mullin, 'A generalization of the singular direct product with application to skew Room squares,' J. Combin. Theory Ser. A 29 (1980), 306-318.

[9] R. C. Mullin, P. J. Schellenberg, S. A. Vanstone and W. D. Wallis, 'On the existence of frames', Discrete Math. 37 (1981), 79-104.

[10] D. K. Ray-Chaudhuri and R. M. Wilson, 'Solution of Kirkman's school-girl problem', Amer. Math. Soc. Proc. Sympos. Pure Math. 19 (1971), 187-204.

[11] R. Rees and D. R. Stinson, 'On resolvable group-divisible designs with block size 3', Ars Combin. 23 (1987), 107-120. 
[12] R. Rees and D. R. Stinson, 'On combinatorial designs with subdesigns', Ann. Discrete Math., to appear.

[13] R. G. Stanton and J. L. Allston, 'A census of values for $g^{(k)}(1,2 ; v)$, Ars Combin. 20 (1985), 203-216.

[14] G. Stern, 'Tripelsysteme mit Untersystemen, ' Arch. Math. 33 (1979), 204-208.

[15] G. Stern and H. Lenz, 'Steiner triple systems with given subspaces; another proof of the Doyen-Wilson theorem', Boll. Un. Mat. Ital. A 17 (1980), 109-114.

[16] R. M. Wilson, 'Constructions and uses of pairwise balanced designs', Math. Centre Tracts 55 (1974), 18-41.

Department of Computer Science

University of Manitoba

Winnipeg

Manitoba R3T 2N2

Canada 